This item was submitted to Loughborough's Research Repository by the author.

Items in Figshare are protected by copyright, with all rights reserved, unless otherwise indicated.

\title{
Myths and methodologies: how loud is the story told by the transcranial magnetic stimulation-evoked silent period?
}

\author{
PLEASE CITE THE PUBLISHED VERSION
}

https://doi.org/10.1113/ep087557

\section{PUBLISHER}

Wiley

VERSION

AM (Accepted Manuscript)

\section{PUBLISHER STATEMENT}

This is the peer reviewed version of the following article: ŠKARABOT, J. ... et al, 2019. Myths and methodologies: how loud is the story told by the transcranial magnetic stimulation-evoked silent period? Experimental Physiology, 104 (5), pp.635-642, which has been published in final form at https://doi.org/10.1113/EP087557. This article may be used for non-commercial purposes in accordance with Wiley Terms and Conditions for Use of Self-Archived Versions.

\section{LICENCE}

CC BY-NC-ND 4.0

\section{REPOSITORY RECORD}

Skarabot, Jakob, Ricardo NO Mesquita, Callum G Brownstein, and Paul Ansdell. 2019. "Myths and Methodologies: How Loud Is the Story Told by the Transcranial Magnetic Stimulation-evoked Silent Period?". Loughborough University. https://hdl.handle.net/2134/14185112.v1. 

(1)

Myths and Methodologies: how loud is the story told by the transcranial magnetic stimulation-evoked silent period?

Jakob Škarabot ${ }^{1}$, Ricardo N O Mesquita ${ }^{2}$, Callum G Brownstein ${ }^{1,3}$ and Paul Ansdell ${ }^{1}$

1 Faculty of Health and Life Sciences, Northumbria University, Newcastle upon Tyne, UK

2 Faculty of Medical and Health Sciences, Edith Cowan University, Perth, Australia

3 Univ Lyon, UJM Saint-Etienne, Laboratoire Interuniversitaire de Biologie de la Motricité, Saint-Étienne, France

Running title: The TMS silent period

Key words: Corticospinal tract, intracortical inhibition, motor evoked potential, motor cortex, transcranial magnetic stimulation

Word count: 3379

References: 46

\section{Corresponding author}

Mr. Paul Ansdell, MSc, BSc

Faculty of Health and Life Sciences

Northumbria University

2 NE1 8ST

3 Newcastle upon Tyne

4 United Kingdom

5 paul.ansdell@northumbria.ac.uk 


\section{$1 \quad$ What is the topic of this review?}

2 The origin, interpretation and methodological constraints of the silent period induced by

3 transcranial magnetic stimulation are reviewed.

\section{$4 \quad$ What advances does it highlight?}

5 The silent period is generated by both cortical and spinal mechanisms. Therefore, it seems

6 inappropriate to preface silent period with 'cortical' unless additional measures are

7 employed. Due to many confounding variables, a standardised approach to the silent period measurement cannot be suggested. Rather, recommendations of best practice are provided based on the available evidence and the context of the research question.

\section{Abstract}

Transcranial magnetic stimulation of the motor cortex evokes a response in the muscle that can be recorded via electromyography (EMG). One component of this response, when elicited during a voluntary contraction, is a period of EMG silence, termed the silent period (SP), which follows a motor evoked potential (MEP). Modulation of SP duration was long thought to reflect the degree of intracortical inhibition. However, the evidence presented in this review suggests that both cortical and spinal mechanisms contribute to generation of the SP, which makes prefacing SP with 'cortical' misleading. Further investigations with multi-methodological approaches, such as TMS-EEG coupling or interaction of TMS with neuroactive drugs, are needed to make such inferences with greater confidence. A multitude of methodological factors can influence SP and thus confound the interpretation of this measure; namely, background muscle activity, instructions given to the participant, stimulus intensity and the size of the MEP preceding the SP, and the 
1 approach to analysis. A systematic understanding of how the confounding factors

2 influence the interpretation of SP is lacking, making standardisation of the methodology

3 difficult to conceptualise. Rather, the methodology should be guided through the lens of

4 the research question and the population studied, ensuring greater reproducibility,

5 repeatability and comparability of datasets. Recommendations are provided for the best 6 practice within a given context of the experimental design. 
Transcranial magnetic stimulation (TMS) over the motor cortex permits the investigation of the corticospinal pathway, the primary conduit in the control of voluntary movement in humans (Lemon, 2008). Evoked responses to TMS in the target muscle can be recorded with surface electromyography (EMG). When applied during a voluntary contraction, two EMG responses are elicited: an excitatory motor evoked potential (MEP), followed immediately by a period of EMG silence before the activity resumes its pre-stimulus level. This period of EMG silence is known as the silent period (SP), typically lasting $\sim 100-300$ ms, and has historically been assumed to reflect intracortical inhibition (Säisänen et al., 2008). Despite the limitations of current methodological approaches, it has been suggested that spinal mechanisms might have a much more prolonged and influential contribution to the SP than previously thought (Yacyshyn, Woo, Price, \& McNeil, 2016). Nevertheless, the relative contributions of supraspinal and spinal factors, as well as the exact underlying mechanisms that modulate SP duration are still subject to considerable debate. Elucidation of these mechanisms is highly meaningful as inhibitory neural systems are essential for the modulation of excitatory input and maintenance of synaptic stability. Whilst the SP is often not the primary outcome variable, it is used as one of the main measures of central nervous system (CNS) inhibition, with the changes in SP being implicated in phenomena such as neurological disease (Nantes et al., 2016), exerciseinduced neuroplasticity (Kidgell, Bonanno, Frazer, Howatson, \& Pearce, 2017) and fatigue (Goodall, Howatson, \& Thomas, 2018).

Furthermore, taking into consideration the current heterogeneity of methodological approaches used to measure this variable, recommendations of best practice should be provided in order to improve clinical utility of SP as a measure of CNS inhibition. The 
1 present review describes current knowledge of mechanisms underpinning the SP; discusses approaches to measuring the SP as well as potential confounding influences on its measurement; and provides recommendations for best practice of SP measurement. The information provided can guide future research and mitigate the heterogeneity which currently exists.

\section{Quantifying the silent period}

The duration of the SP is quantified by the demarcation of SP onset and offset. Historically, the onset of SP has been defined as either the stimulus artefact, MEP onset (relative SP), or MEP offset (absolute SP), as shown in Figure 1. These time points can be assessed visually or using mathematical criteria (based on the characteristics of pre- and/or poststimulus EMG activity; Damron, Dearth, Hoffman, \& Clark, 2008). Similarly, the SP offset, i.e. the point of resumption of pre-stimulus EMG activity, has been defined using criteria such as “ \pm 2 SD of pre-stimulus EMG for at least 100 ms” (Goodall, Ross, \& Romer, 2010), "resumption of pre-stimulus EMG for greater than 50 ms" (Groppa et al., 2012), or by visual inspection (Damron et al., 2008). Regardless of the analytical approach to quantification of the SP duration, the abovementioned methods display excellent reliability and low variability (Damron et al., 2008).

\section{The origin of silent period}

Despite some early indication that SP may be of solely cortical origin (Schnitzler \& Benecke, 1994), a plethora of evidence suggests it is mediated by both cortical and spinal mechanisms (Fuhr, Agostino, \& Hallett, 1991; Inghilleri, Berardelli, Cruccu, \& Manfredi, 
1 1993; Triggs et al., 1993; Wilson, Lockwood, Thickbroom, \& Mastaglia, 1993; Ziemann,

2 Netz, Szelényi, \& Hömberg, 1993). However, the exact contribution of cortical and

3 subcortical mediation of SP, and the mechanism(s) that are responsible for modulation of

$4 \quad$ SP duration in health and disease remain debatable.

5 Early lines of evidence ascribed the first 50-80 ms of SP to a spinal origin, due to the

6 depression of H-reflexes, an electrophysiological measure of Ia axon monosynaptic input to spinal motoneurons (Zehr, 2002), when conditioned by TMS (Fuhr et al., 1991; Ziemann et al., 1993). This H-reflex depression was attributed to motoneuron afterhyperpolarisation, Renshaw cell inhibition or disynaptic facilitation via Ia inhibitory interneurons (Cantello, Gianelli, Civardi, \& Mutani, 1992; Fuhr et al., 1991; Triggs et al., 1993; Ziemann et al., 1993). The later part of the SP is thought to be mediated by intracortical inhibition, and the longer-lasting activity of $\gamma$-aminobutyric acid (GABA; McDonnell et al., 2006). These conclusions were based on a prolonged SP after administration of а GABAв receptor agonist (Siebner, Dressnandt, Auer, \& Conrad, 1998) and a GABA reuptake inhibitor (Werhahn, Kunesch, Noachtar, Benecke, \& Classen, 1999). Thus, it has long been interpreted that any change in SP duration is a result of modulation in activity of intracortical inhibitory neurons and hence SP is often prefaced by the word cortical.

Notably, the behaviour of the H-reflex, which has primarily been used to infer the spinal contribution to the SP, might be confounded by the marked influence of presynaptic inhibition (Zehr, 2002). Recent evidence obtained using direct subcortical activation of corticospinal axons, which is devoid of classical presynaptic influence (Nielsen \& Petersen, 1994) and thus arguably a more robust technique to assess motoneuron excitability, suggested that the spinal motoneuronal component of the SP might be 
1 substantially greater than previously demonstrated (Yacyshyn et al., 2016). Specifically, when electrical stimulation of the cervicomedullary junction was delivered during a $\sim 200$ ms SP, the index of motoneuron excitability (CMEP) was depressed throughout the 150 ms investigated (Yacyshyn et al., 2016). It should be noted, however, that Yacyshyn and colleagues compared CMEPs elicited during a contraction to those elicited during the SP (Yacyshyn et al., 2016), thus not comparing CMEPs at the same level of neural drive. Whilst within-condition results were not reported, it appears that CMEPs were reduced in size in the first $\sim 80-90 \mathrm{~ms}$, after which they plateaued. As such, the prolonged reduction in CMEPs during SP might reflect the differing magnitude of facilitation stemming from reduced neural drive, rather than the extent of spinal inhibition, which appears to be limited to the initial $\sim 80-90$ milliseconds. This notion is also supported by findings that the SP following CMEPs tend to be shorter than those following MEPs (Inghilleri et al., 1993), though those responses are also accompanied by differing twitch amplitudes (see subsequent paragraph). Alternatively, the prolonged reduction in the size of the CMEP evoked in the SP could be a result of disfacilitation mediated by withdrawal of cortical input. Based on complete suppression of responses to magnetic stimulation, but not double electrical stimuli of the motor cortex when conditioned by TMS at rest, a cortical inhibitory mechanism has been suggested to contribute to the SP at $\geq 100 \mathrm{~ms}$ (Inghilleri et al., 1993). A combined approach eliciting CMEPs and cortical stimuli during the SP, whilst comparing unconditioned responses at rest, is warranted to delineate a more exact contribution of spinal and cortical mechanisms to the SP.

The finding of reduced motoneuron excitability during SP elicited at $25 \%$ of maximal voluntary contraction (MVC) was attributed to disfacilitation mediated by reduced spindle discharge, stemming from muscle spindle unloading and increased Ib inhibition via Golgi tendon organs as a result of a large TMS-induced twitch (Yacyshyn et al., 2016). 
1 However, it not clear how this hypothesis would apply to the SPs elicited during MVCs, where TMS-induced twitches are significantly smaller compared to submaximal contractions (Todd, Taylor, \& Gandevia, 2016), making tendon organs less likely to discharge and muscle spindles to be unloaded to a lesser extent. It seems more likely that extra firing of muscle spindles would occur during the muscle relaxation phase at the time of the SP as a result of muscle lengthening (Butler, Petersen, Herbert, Gandevia, \& Taylor, 2012; Yacyshyn, Nettleton, Power, Jakobi, \& McNeil, 2017). On this note, it is worth noting that the SP can also be interrupted by small bursts of EMG activity or low-level of EMG activity before recommencement of voluntary EMG (Butler et al., 2012). Although this has been postulated to arise from ipsilateral cortical or subcortical structures (Holmgren, Larsson, \& Pedersen, 1990), it is more likely mediated by increased firing rate of muscle spindles upon muscle lengthening as a result of muscle relaxation (Figure 2; Butler et al., 2012). Whilst spinal inhibition cannot be discounted as partly contributing to the later portion of SP, it seems insufficient to supress muscle spindle excitation during SP when these EMG bursts occur. Equally, intracortical inhibition in the later part of the SP might be insufficient to supress the late low-level EMG activity, particularly if the latter is used to mark the end of SP.

Collectively, the aforementioned data suggests that SP is a combination of spinal and cortical mechanisms (Figure 2). Intracortical inhibition is likely to contribute to the SP throughout its duration due to the long-lasting activity of cortical GABAergic neurons (Krnjević, Randić, \& Straughan, 1966). Conversely, whilst the twitch-related mechanisms could vary with twitch characteristics (the size and duration of the twitch influenced by the stimulus intensity; Todd et al., 2016) as demonstrated in Figure 2, the contribution of disynaptic disfacilitation is likely restricted to the initial part of SP due to short-lasting activity of GABAergic interneurons at the spinal cord (Inghilleri et al., 1993). Furthermore, 
1 recurrent inhibition stemming from the onset of a large excitatory response is likely

restricted to the initial $\sim 40 \mathrm{~ms}$ after the evoked response (Bussel \& Pierrot-Deseilligny, 1977). Similarly, afterhyperpolarisation of motoneurons will depend on their firing frequency $(\sim 20-50 \mathrm{~Hz})$, again restricting the contribution of this mechanism to the initial 40 ms following a MEP (Enoka \& Fuglevand, 2001). Due to these multiple concurrent mechanisms, it seems misleading to preface SP with cortical. We suggest that the SP should not be prefaced with an adjective such as cortical and could simply be referred to as the 'TMS-evoked SP' or just 'SP' (when used within the context of TMS) to avoid confusion or inaccuracy. In the cases when the SP is longer than the duration of the muscle twitch, the inferences about the cortically-mediated changes are likely valid. Despite the longer lasting cortical component, it remains unclear whether the temporal contribution of spinal mediators of SP is static. Indeed, many of the postulated mechanisms thought to contribute to the spinal component of the SP, such as disynaptic disfacilitation, recurrent inhibition, and afferent firing from muscle receptors could conceivably be altered in certain movement disorders (Nalbantoglu, Battal, Kiziltan, Akalin, \& Kiziltan, 2016), spasticity (Mukherjee \& Chakravarty, 2010), or by fatiguing exercise (Macefield, Hagbarth, Gorman, Gandevia, \& Burke, 1991). Furthermore, it has been shown that motoneuron excitability at $100 \mathrm{~ms}$ into the SP is reduced during fatiguing exercise (Finn, Rouffet, Kennedy, Green, \& Taylor, 2018), whilst the SP itself might be prolonged as a consequence of fatigue (Goodall et al., 2018). It is possible that this increase in SP duration and decrease in motoneuron excitability are both caused by increased inhibition of spinal motoneurons. However, this requires a systematic investigation in the future. Perhaps conclusions with greater accuracy about the cortical inhibitory mechanisms could be made from the SP with concurrent investigation of spinal motoneurons excitability and inhibition and/or by employing a multi-methodological approach, such as coupling TMS 
1 with electroencephalography, neuroactive drugs or magnetic resonance spectroscopy

2 (Ziemann, 2011).

3

4

\section{Confounding factors influencing silent period interpretation, and} recommendations for best practice

It is well established that the SP can be influenced by a number of variables involved in the delivery of TMS. At present, considerable heterogeneity exists in the methodological approach to SP assessment, thus precluding comparability between studies, and potentially inferences that can be made. Limitations inherent to measuring and analysing the SP preclude the recommendation of a single approach to SP assessment. Rather, researchers should aim to make informed decisions on the best approach to measure SP based on the current evidence, the research question they pose (e.g. exercise or pharmacological interventions, assessment of disturbances in motor system function in health and disease), and the population under study (e.g. clinical or athletic populations). The confounding methodological factors to eliciting and measuring the SP are discussed below, along with recommendations about the best approaches in a given context (summarised in Table 1).

\section{Background muscle activity and participant instructions}

By definition, the SP must be evoked during muscle contraction. Depending on the research question and study design, researchers have chosen to induce SPs at a wide range of contraction intensities, ranging from $10 \%$ (Goodall et al. 2018) to $100 \%$ MVC (Mira et al., 2017). Whilst previous data is equivocal as to whether contraction intensity 
and the associated background EMG affects SP duration (Säisänen et al., 2008; Taylor, Allen, Butler, \& Gandevia, 1997; Wilson et al., 1993), recent findings suggest the SP duration decreases with an increase in force output (Matsugi, 2019). The author attributed these findings to the role of inhibitory mechanisms in force control. However, modulation of SP with contraction strength may partly be due to different characteristics of the muscle twitch. The relationship between contraction strength, muscle twitch characteristics and their effect on SP duration warrants further investigation. Regardless, caution should be taken when comparing durations of SP that are not elicited at the same level of neural drive. Säisänen et al (2008) demonstrated that greater contraction intensities (40-60\% MVC) produce the lowest coefficients of variation. This is possibly due to a reduction in measurement error owing to clearer identification of SP offset with higher background EMG activity. Therefore, measuring the SP at intensities equating to $40-60 \%$ of maximal neural drive (measured, for instance, using a percentage of maximum EMG) seems favourable. However, in clinical populations it could be challenging to accurately perform sufficiently steady muscle contractions with this intensity and therefore lower contraction intensities might be more suitable. Indeed, evidence suggests that if TMS is not delivered on a steady plateau in force, the resultant TMS-evoked response is altered (Gruet, Temesi, Rupp, Millet, \& Verges, 2013).

Similarly, the post-stimulus behaviour influences SP duration. A "rebound hard and fast" instruction given to the participant appears to shorten the SP, potentially due to increased neural drive overcoming late SP inhibitory influences, whereas a "relax" instruction prolonged the resumption of EMG activity for the opposite reason (Mathis, De Quervain, \& Hess, 1998). We recommend that instructing participants to rebound after the stimulus 
1 will more likely give a valid indication of inhibition for a given neural drive and mitigate any influence of post-stimulus differences in muscle activity.

\section{Stimulus intensity and MEP amplitude}

The most influential confounding factor on SP duration appears to be the intensity of TMS used to evoke the MEP and SP (Inghilleri et al., 1993; Säisänen et al., 2008; Wilson et al., 1993), with longer SPs exhibited at higher intensities of stimulation until a plateau occurring at very high stimulus intensities (Kimiskidis et al., 2005). This relationship might be due to the influence that the preceding MEP, which inherently increase with stimulus intensity, have on SP duration (Orth \& Rothwell, 2004). Therefore, Orth \& Rothwell (2004) recommended calculating a SP:MEP ratio to reduce between-subject variability and reflect a balance between excitatory and inhibitory mechanisms. It is suggested that if MEP size increases without concurrent lengthening of the SP, this would correspond to a decrease in the inhibitory influence within the CNS, with the inverse relationship reflecting increased inhibition (Orth \& Rothwell, 2004).

It is suggested that constructing a stimulus-response curve provides the most comprehensive measure of SP and mitigates any potential influence of alterations in motor threshold on changes in SP (Kimiskidis et al., 2005). This approach might be suitable when combined with a lower contraction intensity, and when there is no timeconstraint associated with the assessment. However, many studies require the SP to be captured in a timely fashion (e.g. during or following fatiguing exercise), in which case this more time-consuming approach would be unsuitable. In these instances, studies often measure the SP at an intensity relative to motor threshold (Goodall et al., 2018), defined as stimulus intensity that produces a reliable MEP of minimal amplitude in the target 
muscle (Rossini et al., 2015). The limitation with this approach is that the motor threshold and SP are thought to be physiologically distinct and might be modulated differently (Kimiskidis et al., 2005), with SP potentially having a lower threshold and occurring without an MEP (Wassermann et al., 1993). As such, using the approach where responses are standardised to a single value relative to motor threshold could lead to inaccurate interpretation if changes in motor threshold occur. One approach which could circumvent this issue is to construct the SP recruitment curve pre-exercise, then use a fixed stimulus intensity on the ascending limb of the recruitment curve when eliciting SP post exercise (Fritz, Braune, Pylatiuk, \& Pohl, 1997). Kimiskidis et al (2005) suggested that the intensity above threshold corresponding to the plateau of the sigmoidal curve represents the intensity at which inhibitory influences are maximised. However, at these high stimulus intensities, the SP duration would not lie on the ascending arm of this relationship, thus becoming saturated, and less likely to exhibit changes in response to an intervention or disease state. As such, we suggest that stimulus intensities that elicit the SP on the ascending limb of the recruitment curve are favourable.

\section{Analysis of the silent period}

As previously mentioned, a number of reference points have been used to define the SP onset (Figure 1), with potential confounds arising from each of them. Since the mechanisms that are involved in the generation of the SP are prompted after the stimulus, the stimulus artefact seems to be the most suitable standardised reference point for SP onset. It should be noted that when stimulus artefact is used to define SP onset, issues can arise in populations exhibiting changes in evoked response latencies, such as the elderly (Opie, Cirillo, \& Semmler, 2018). However, it is unknown whether longer MEP latencies are necessarily associated with a longer delay for the commencement of inhibitory 
1 mechanisms. Similarly, caution should be taken when defining SP onset as MEP offset in cases where slowing of neuromuscular transmission is expected (e.g fatigue; Gandevia et al., 2013), since an increase in MEP duration does not necessarily delay the commencement of inhibitory behaviour. Thus, it might be appropriate to quantify and report SP from all three time points (stimulus artefact, MEP onset, and MEP offset), and to analyse and interpret the SP with any confounding influences in mind (e.g. MEP latency and duration).

SP offset also presents challenges, particularly when small 'bursts' of low-level EMG activity appear during the SP. The issue then becomes whether to set SP offset as the 'burst' or the resumption of EMG following the second suppression. As discussed earlier, this low-level EMG is likely reflexive in origin and does not reflect the suppression of voluntary EMG (Butler et al., 2012). Thus, careful visual inspection or using a larger criterion for SP offset (i.e. \pm 2 SD of pre-stimulus EMG) will likely avoid this issue. Since the number of examiners can affect reproducibility (Fritz et al., 1997), using a mathematical criterion (e.g. \pm 2 SD of pre-stimulus EMG) is likely to be more reliable and is thus recommended.

A pertinent question with regards to the analysis of SP is also the number of trials required to obtain a representative mean value of the SP duration. For MEPs, it seems that at least 20 trials are needed to obtain an accurate estimate of corticospinal excitability during muscle contraction (Brownstein et al., 2018). The SP tend to be less variable than MEPs (Säisänen et al., 2008), which could suggest that fewer trials are needed. Six to eight trials have been recommended previously (Rossini et al., 2015). However, future research should attempt to provide clarity on this issue. 


\section{Summary and future directions}

4 The evidence presented in this review suggests the SP is mediated by both spinal and

5 cortical mechanisms with the relative contribution of each currently debatable.

6 Therefore, using adjectives in relation to the SP origin seems inappropriate. It remains

7 unclear whether the spinal component of the SP is invariable, and to what extent spinal

8 and cortical components are inter-related. Future research should explore this further in

9 a systematic manner. A multi-methodological approach would allow mechanistic inferences about the modulation of SP to be inferred with a higher degree of confidence.

The silent period is influenced by a myriad of confounding factors ranging from background muscle activity, instructions given to the participant, stimulus intensity, the size of MEP preceding SP and the approach to analysis. In order to facilitate greater clinical utility of SP, it is important to better understand its validity as a measure of CNS inhibition, reliability and the factors that might confound its interpretation. Alterations of known confounding factors should be investigated in a systematic manner, perhaps in conjunction with the use of pharmacological agents that are known to alter inhibitory synaptic input in the CNS, to delineate the reliability and sensitivity of different methodological approaches. 


\section{References}

Brownstein, C., Ansdell, P., Škarabot, J., Howatson, G., Goodall, S., \& Thomas, K. (2018). An optimal protocol for measurement of corticospinal excitability, short intracortical inhibition and intracortical facilitation in the rectus femoris. Journal of the Neurological Sciences. http://doi.org/10.1016/j.jns.2018.09.001

Bussel, B., \& Pierrot-Deseilligny, E. (1977). Inhibition of human motoneurons, probably of Renshaw origin, elicited by an orthodromic motor discharge. The Journal of Physiology, 269(2), 319-39. http://doi.org/10.1113/jphysiol.1977.sp011904

Butler, J. E., Petersen, N. C., Herbert, R. D., Gandevia, S. C., \& Taylor, J. L. (2012). Origin of the low-level EMG during the silent period following transcranial magnetic stimulation. Clinical Neurophysiology, 123(7), 1409-1414. http://doi.org/10.1016/j.clinph.2011.11.034

Cantello, R., Gianelli, M., Civardi, C., \& Mutani, R. (1992). Magnetic brain stimulation: the silent period after the motor evoked potential. Neurology, 42(10), 1951-1959.

Damron, L. A., Dearth, D. J., Hoffman, R. L., \& Clark, B. C. (2008). Quantification of the corticospinal silent period evoked via transcranial magnetic stimulation. Journal of Neuroscience Methods, 173(1), 121-128. http://doi.org/10.1016/j.jneumeth.2008.06.001

Enoka, R. M., \& Fuglevand, A. J. (2001). Motor unit physiology: some unresolved issues. Muscle \& Nerve, 24(1), 4-17. http://doi.org/10.1002/10974598(200101)24:1<4::AID-MUS13>3.0.C0;2-F

Finn, H. T., Rouffet, D. M., Kennedy, D. S., Green, S., \& Taylor, J. L. (2018). Motoneuron excitability of the quadriceps decreases during a fatiguing submaximal isometric contraction. Journal of Applied Physiology (Bethesda, Md. : 1985). http://doi.org/10.1152/japplphysiol.00739.2017

Fritz, C., Braune, H. J., Pylatiuk, C., \& Pohl, M. (1997). Silent period following transcranial magnetic stimulation: a study of intra- and inter-examiner reliability. Electroencephalography and Clinical Neurophysiology, 105(3), 235-40.

Fuhr, P., Agostino, R., \& Hallett, M. (1991). Spinal motor neuron excitability during the silent period after cortical stimulation. Electroencephalography and Clinical Neurophysiology, 81(4), 257-62.

Gandevia, S. C., McNeil, C. J., Carroll, T. J., \& Taylor, J. L. (2013). Twitch interpolation: superimposed twitches decline progressively during a tetanic contraction of human adductor pollicis. The Journal of Physiology, 591(5), 1373-83. http://doi.org/10.1113/jphysiol.2012.248989

Goodall, S., Howatson, G., \& Thomas, K. (2018). Modulation of specific inhibitory networks in fatigued locomotor muscles of healthy males. Experimental Brain Research, 236(2), 463-473. http://doi.org/10.1007/s00221-017-5142-x

Goodall, S., Ross, E. Z., \& Romer, L. M. (2010). Effect of graded hypoxia on supraspinal contributions to fatigue with unilateral knee-extensor contractions. Journal of 
Applied Physiology, 109(6), 1842-1851.

http://doi.org/10.1152/japplphysiol.00458.2010

Groppa, S., Oliviero, A., Eisen, A., Quartarone, A., Cohen, L. G., Mall, V., ... Siebner, H. R. (2012). A practical guide to diagnostic transcranial magnetic stimulation: report of an IFCN committee. Clinical Neurophysiology, 123(5), 858-82. http://doi.org/10.1016/j.clinph.2012.01.010

Gruet, M., Temesi, J., Rupp, T., Millet, G. Y., \& Verges, S. (2013). Effect of different approaches to target force on transcranial magnetic stimulation responses. Muscle \& Nerve, 48(3), 430-2. http://doi.org/10.1002/mus.23786

Holmgren, H., Larsson, L. E., \& Pedersen, S. (1990). Late muscular responses to transcranial cortical stimulation in man. Electroencephalography and Clinical Neurophysiology, 75(3), 161-72.

Inghilleri, M., Berardelli, A., Cruccu, G., \& Manfredi, M. (1993). Silent period evoked by transcranial stimulation of the human cortex and cervicomedullary junction. The Journal of Physiology, 466(1), 521-534. http://doi.org/10.1113/jphysiol.1993.sp019732

Kidgell, D. J., Bonanno, D. R., Frazer, A. K., Howatson, G., \& Pearce, A. J. (2017). Corticospinal responses following strength training: a systematic review and metaanalysis. European Journal of Neuroscience, 46(11), 2648-2661. http://doi.org/10.1111/ejn.13710

Kimiskidis, V. K., Papagiannopoulos, S., Sotirakoglou, K., Kazis, D. A., Kazis, A., \& Mills, K. R. (2005). Silent period to transcranial magnetic stimulation: Construction and properties of stimulus-response curves in healthy volunteers. Experimental Brain Research, 163(1), 21-31. http://doi.org/10.1007/s00221-004-2134-4

Krnjević, K., Randić, M., \& Straughan, D. W. (1966). An inhibitory process in the cerebral cortex. The Journal of Physiology, 184(1), 16-48. http://doi.org/doi.org/10.1113/jphysiol.1966.sp007902

Lemon, R. N. (2008). Descending Pathways in Motor Control. Annual Review of Neuroscience, 31(1), 195-218. http://doi.org/10.1146/annurev.neuro.31.060407.125547

Macefield, G., Hagbarth, K. E., Gorman, R., Gandevia, S. C., \& Burke, D. (1991). Decline in spindle support to alpha-motoneurones during sustained voluntary contractions. The Journal of Physiology, 440, 497-512. http://doi.org/10.1113/jphysiol.1991.sp018721

Mathis, J., De Quervain, D., \& Hess, C. W. (1998). Dependence of the transcranially induced silent period on the "instruction set" and the individual reaction time. Electroencephalography and Clinical Neurophysiology - Electromyography and Motor Control, 109(5), 426-435. http://doi.org/10.1016/S0924-980X(98)00042-3

Matsugi, A. (2019). Changes in the cortical silent period during force control. Somatosensory \& Motor Research, 1-6. http://doi.org/10.1080/08990220.2018.1563536 
McDonnell, M. N., Orekhov, Y., \& Ziemann, U. (2006). The role of GABAB receptors in intracortical inhibition in the human motor cortex. Experimental Brain Research, 173(1), 86-93. http://doi.org/10.1007/s00221-006-0365-2

Mira, J., Lapole, T., Souron, R., Messonnier, L., Millet, G. Y., \& Rupp, T. (2017). Cortical voluntary activation testing methodology impacts central fatigue. European Journal of Applied Physiology, 117(9), 1845-1857. http://doi.org/10.1007/s00421-0173678-x

Mukherjee, A., \& Chakravarty, A. (2010). Spasticity mechanisms - for the clinician. Frontiers in Neurology, 1, 149. http://doi.org/10.3389/fneur.2010.00149

Nalbantoglu, M., Battal, H., Kiziltan, M. E., Akalin, M. A., \& Kiziltan, G. (2016). Stiff Person Syndrome with Pyramidal Signs. Noro Psikiyatri Arsivi, 53(2), 188-190. http://doi.org/10.5152/npa.2015.11310

Nantes, J. C., Zhong, J., Holmes, S. A., Narayanan, S., Lapierre, Y., \& Koski, L. (2016). Cortical Damage and Disability in Multiple Sclerosis: Relation to Intracortical Inhibition and Facilitation. Brain Stimulation, 9(4), 566-573. http://doi.org/10.1016/j.brs.2016.01.003

Nielsen, J., \& Petersen, N. (1994). Is presynaptic inhibition distributed to corticospinal fibres in man? The Journal of Physiology, 477(Pt 1), 47-58. http://doi.org/10.1113/jphysiol.1994.sp020170

Opie, G. M., Cirillo, J., \& Semmler, J. G. (2018). Age-related changes in late I-waves influence motor cortex plasticity induction in older adults. Journal of Physiology, 596(13), 2597-2609. http://doi.org/10.1113/JP274641

Orth, M., \& Rothwell, J. . (2004). The cortical silent period: intrinsic variability and relation to the waveform of the transcranial magnetic stimulation pulse. Clinical Neurophysiology, 115(5), 1076-1082. http://doi.org/10.1016/j.clinph.2003.12.025

Rossini, P. M., Burke, D., Chen, R., Cohen, L. G., Daskalakis, Z., Di Iorio, R., ... Ziemann, U. (2015). Non-invasive electrical and magnetic stimulation of the brain, spinal cord, roots and peripheral nerves: Basic principles and procedures for routine clinical and research application. An updated report from an I.F.C.N. Committee. Clinical Neurophysiology, 126(6), 1071-1107. http://doi.org/10.1016/j.clinph.2015.02.001

Säisänen, L., Pirinen, E., Teitti, S., Könönen, M., Julkunen, P., Määttä, S., \& Karhu, J. (2008). Factors influencing cortical silent period: Optimized stimulus location, intensity and muscle contraction. Journal of Neuroscience Methods, 169(1), 231-238. http://doi.org/10.1016/j.jneumeth.2007.12.005

Schnitzler, A., \& Benecke, R. (1994). The silent period after transcranial magnetic stimulation is of exclusive cortical origin: evidence from isolated cortical ischemic lesions in man. Neuroscience Letters, 180(1), 41-5. http://doi.org/10.1016/03043940(94)90909-1

Siebner, H. R., Dressnandt, J., Auer, C., \& Conrad, B. (1998). Continuous intrathecal baclofen infusions induced a marked increase of the transcranially evoked silent period in a patient with generalized dystonia. Muscle \& Nerve, 21(9), 1209-12. 
http://doi.org/10.1002/(SICI)1097-4598(199809)21:9<1209::AID-

MUS15>3.0.CO;2-M

Taylor, J. L., Allen, G. M., Butler, J. E., \& Gandevia, S. C. (1997). Effect of contraction strength on responses in biceps brachii and adductor pollicis to transcranial magnetic stimulation. Experimental Brain Research, 117(3), 472-478. http://doi.org/10.1007/s002210050243

Todd, G., Taylor, J. L., \& Gandevia, S. C. (2016). Measurement of voluntary activation based on transcranial magnetic stimulation over the motor cortex. Journal of Applied Physiology, 121(3), 678-686. http://doi.org/10.1152/japplphysiol.00293.2016

Triggs, W. J., Cros, D., Macdonell, R. A., Chiappa, K. H., Fang, J., \& Day, B. J. (1993). Cortical and spinal motor excitability during the transcranial magnetic stimulation silent period in humans. Brain Research, 628(1-2), 39-48. http://doi.org/10.1016/00068993(93)90935-G

Wassermann, E. M., Pascual-Leone, A., Valls-Solé, J., Toro, C., Cohen, L. G., \& Hallett, M. (1993). Topography of the inhibitory and excitatory responses to transcranial magnetic stimulation in a hand muscle. Electroencephalography and Clinical Neurophysiology, 89(6), 424-33.

Werhahn, K. J., Kunesch, E., Noachtar, S., Benecke, R., \& Classen, J. (1999). Differential effects on motorcortical inhibition induced by blockade of GABA uptake in humans. The Journal of Physiology, 591-7. http://doi.org/10.1111/j.1469-7793.1999.0591t.x

Wilson, S. A., Lockwood, R. J., Thickbroom, G. W., \& Mastaglia, F. L. (1993). The muscle silent period following transcranial magnetic cortical stimulation. Journal of the Neurological Sciences, 114(2), 216-22. Retrieved from http://www.ncbi.nlm.nih.gov/pubmed/8445404

Yacyshyn, A. F., Nettleton, J., Power, G. A., Jakobi, J. M., \& McNeil, C. J. (2017). The effect of muscle length on transcranial magnetic stimulation-induced relaxation rate in the plantar flexors. Physiological Reports, 5(18), e13442. http://doi.org/10.14814/phy2.13442

Yacyshyn, A. F., Woo, E. J., Price, M. C., \& McNeil, C. J. (2016). Motoneuron responsiveness to corticospinal tract stimulation during the silent period induced by transcranial magnetic stimulation. Experimental Brain Research, 234(12), 3457-3463. http://doi.org/10.1007/s00221-016-4742-1

Zehr, E. P. (2002). Considerations for use of the Hoffmann reflex in exercise studies. European Journal of Applied Physiology, 86(6), 455-468. http://doi.org/10.1007/s00421-002-0577-5

Ziemann, U. (2011). Transcranial Magnetic Stimulation at the Interface with Other Techniques. The Neuroscientist, 17(4), 368-381. http://doi.org/10.1177/1073858410390225

Ziemann, U., Netz, J., Szelényi, A., \& Hömberg, V. (1993). Spinal and supraspinal mechanisms contribute to the silent period in the contracting soleus muscle after 
1 transcranial magnetic stimulation of human motor cortex. Neuroscience Letters,

2 156(1-2), 167-171. http://doi.org/10.1016/0304-3940(93)90464-V

3 


\begin{tabular}{|c|c|c|c|c|c|c|}
\hline & Background activity & Stimulus intensity & $\begin{array}{c}\text { Subject } \\
\text { instruction }\end{array}$ & Analysis - SP onset & $\begin{array}{c}\text { Analysis - SP } \\
\text { offset }\end{array}$ & $\begin{array}{c}\text { Number } \\
\text { of stimuli }\end{array}$ \\
\hline Best practice & $40-60 \% \mathrm{MVC} / \mathrm{EMG} \max$ & Construct SP recruitment curve & $\begin{array}{l}\text { "Rebound after } \\
\text { the stimulus" }\end{array}$ & $\begin{array}{l}\text { Stimulus artefact, } \\
\text { MEP onset, MEP offset } \\
\rightarrow \text { compare all } 3\end{array}$ & $\begin{array}{l} \pm 2 \text { SD of pre- } \\
\text { stimulus EMG } \\
\text { activity }\end{array}$ & $>6$ \\
\hline Alternative & $<40 \% \mathrm{MVC} / \mathrm{EMG} \max$ & $\begin{array}{c}\text { Fixed intensity on the ascending limb } \\
\text { of SP recruitment curve }\end{array}$ & - & Stimulus artefact & - & - \\
\hline $\begin{array}{l}\text { Reason for } \\
\text { alternative }\end{array}$ & $\begin{array}{c}\text { Inability of subjects } \\
\text { to maintain a sufficiently steady } \\
\text { force output at higher contraction } \\
\text { strengths }\end{array}$ & Time constraint & & $\begin{array}{l}\text { Neuromuscular transmission } \\
\text { or MEP latency not } \\
\text { expected to change }\end{array}$ & & \\
\hline
\end{tabular}




\section{Figure captions}

2 Figure 1. Different types of silent periods (SP) depending on the definition of its onset. The point of stimulus, MEP onset, MEP offset and the point of resumption of voluntary EMG are noted by the vertical lines. The SP durations corresponding to each SP type are noted in this example response recorded from the tibialis anterior muscle.

Figure 2. Mechanisms contributing to generation of the silent period. A typical mechanical response to transcranial magnetic stimulation along with electromyographic response of the tibialis anterior muscle including background voluntary EMG activity, motor evoked potential (MEP) and the period of EMG silence. Responses are shown at 10 and $100 \%$ of maximal voluntary contraction (MVC) strength. The right panel is the focused version of the corresponding panel on the left. The approximate temporal contribution of each mechanism to the silent period is noted. The red circle in the upper right panel denotes reflex activity during the silent period as a result of muscle relaxation following stimulation. $\mathrm{TMS}=$ transcranial magnetic stimulation, $\mathrm{AHP}=$ after hyperpolarisation, $\mathrm{RI}=$ recurrent inhibition, $\mathrm{DiDF}=$ disynaptic disfacilitation. 


\section{AKCNOWLEDGMENTS}

The authors express their gratitude to Prof. Janet Taylor and Dr. Stuart Goodall for constructive comments on an earlier draft version of the manuscript and continuing discussion throughout the writing process.

\section{COMPETING INTERESTS}

The authors declare no conflict of interest, financial or otherwise.

\section{AUTHOR CONTRIBUTIONS}

All authors conceived and designed the work; J.Š. prepared figures and tables; all authors drafted the manuscript, edited and revised the manuscript, approved the final version of manuscript and agree to be accountable for all aspects of the work in ensuring that questions related to the accuracy and integrity of any part of the work are appropriately investigated and resolved. All persons designated as author qualify for authorship, and all those who qualify for authorship are listed. 
Point of MEP stimulus onset

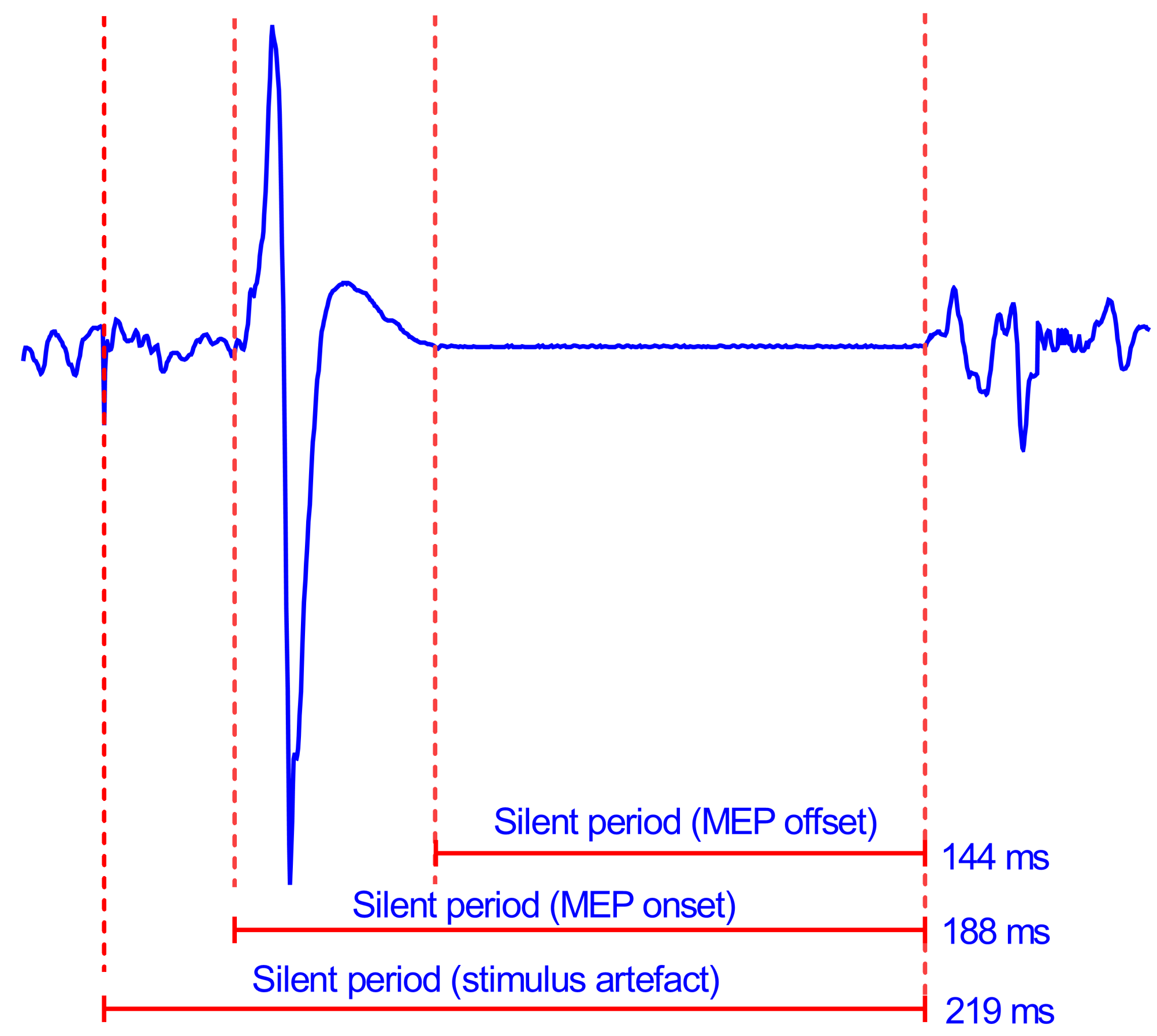

Resumption of voluntary EMG
MEP

offset 
